\title{
Article/Artigo
}

\section{In-house ELISA method to analyze anti-Trypanosoma cruzi IgG reactivity for differential diagnosis and evaluation of Chagas disease morbidity}

\author{
Método de ELISA in-house na análise da reatividade de IgG anti-Trypanosoma cruzi para o \\ diagnóstico diferencial e avaliação da morbidade da doença de Chagas
}

\begin{abstract}
Lilian da Silva Santos ${ }^{1}$, Rosália Morais Torres ${ }^{2}$, Girley Francisco Machado-de-Assis ${ }^{1}$, Maria Terezinha Bahia ${ }^{1,3}$, Helen Rodrigues Martins ${ }^{1,4}$, Andréa Teixeira-Carvalho ${ }^{5}$, Jordana Grazziela Alves Coelho-dos-Reis ${ }^{6}$, Pedro Albajar-Viñas ${ }^{7}$, Olindo Assis Martins-Filho ${ }^{5}$ and Marta de Lana ${ }^{1,8}$
\end{abstract}

\begin{abstract}
Introduction: The goal was to develop an in-house serological method with high specificity and sensitivity for diagnosis and monitoring of Chagas disease morbidity. Methods: With this purpose, the reactivities of anti-T. cruzi IgG and subclasses were tested in successive serum dilutions of patients from Berilo municipality, Jequitinhonha Valley, Minas Gerais, Brazil. The performance of the in-house ELISA was also evaluated in samples from other relevant infectious diseases, including HIV, hepatitis C (HCV), syphilis (SYP), visceral leishmaniasis (VL), and American tegumentary leishmaniasis (ATL), and noninfected controls (NI). Further analysis was performed to evaluate the applicability of this in-house methodology for monitoring Chagas disease morbidity into three groups of patients: indeterminate (IND), cardiac (CARD), and digestive/mixed (DIG/Mix), based on their clinical status. Results: The analysis of total IgG reactivity at serum dilution 1:40 was an excellent approach to Chagas disease diagnosis (100\% sensitivity and specificity). The analysis of IgG subclasses showed cross-reactivity, mainly with NI, VL, and ATL, at all selected serum dilutions. Based on the data analysis, the IND group displayed higher IgG3 levels and the DIG/Mix group presented higher levels of total IgG as compared with the IND and CARD groups. Conclusions: These findings demonstrated that methodology presents promising applicability in the analysis of anti-T. cruzi IgG reactivity for the differential diagnosis and evaluation of Chagas disease morbidity.
\end{abstract}

Keywords: In-house ELISA. IgG subclasses. Differential Diagnosis. Chagas Disease Morbidity.

\section{RESUMO}

Introdução: O objetivo foi desenvolver um método sorológico in-house de alta especificidade e sensibilidade para diagnosticar e monitorar a morbidade da doença de Chagas. Métodos: Para tal, a reatividade sorológica de IgG e subclasses foi testada em soros de pacientes chagásicos de Berilo, Vale do Jequitinhonha/MG/Brasil. A reatividade sorológica foi também avaliada em amostras de pacientes com outras doenças infecto-contagiosas relevantes, incluindo o HIV, vírus da hepatite C (VHC), sífilis (SYP), leishmaniose visceral (LV), leishmaniose tegumentar americana (LTA) e controles não infectados (NI) para verificar o desempenho do método. Outras análises foram feitas para avaliar a aplicabilidade desta metodologia no monitoramento da morbidade da doença de Chagas. Com este propósito os pacientes com doença de Chagas foram anteriormente classificados em três grupos: indeterminados (IND), cardíacos (CARD) e digestivos/mistos (DIG/Mis) conforme seu estado clínico. Resultados: A análise da reatividade sorológica de IgG total na diluição 1:40 mostrou ser uma abordagem importante no diagnóstico da doença de Chagas (100\% de sensibilidade e especificidade e ausência de reação cruzada com as demais infecções). A análise das subclasses de IgG mostrou reação cruzada principalmente com NI, LV e LTA em todas as diluições. O grupo IND apresentou a maior reatividade para IgG3 e o grupo DIG/Mis apresentou nível mais elevado de IgG se comparados aos grupos IND e CARD. Conclusões: Estes achados demonstram que o método de ELISA in-house apresenta uma promissora aplicabilidade no diagnóstico diferencial e na avaliação da morbidade da doença de Chagas.

Palavras-chaves: In-house ELISA. Subclasses de IgG. Diagnóstico diferencial. Morbidade da doença de Chagas.

1. Núcleo de Pesquisas em Ciências Biológicas, Universidade Federal de Ouro Preto, Ouro Preto, MG. 2. Departamento de Clínica Médica, Universidade Federal de Minas Gerais, Belo Horizonte, MG. 3. Departamento de Ciências Biológicas, Universidade Federal de Ouro Preto, Ouro Preto, MG. 4. Departamento de Farmácia, Universidade Federal do Vale do Jequitinhonha e Mucuri, Diamantina, MG. 5. Laboratório de Biomarcadores de Diagnóstico e Monitoração, Centro de Pesquisas René Rachou, Fundação Oswaldo Cruz, Belo Horizonte, MG. 6. Department of Microbiology \& Immunology, Drexel University College of Medicine, Doylestown, PA, USA. 7. Laboratório de Doenças Parasitárias, Fundação Oswaldo Cruz, Rio de Janeiro, RJ. 8. Departamento de Análises Clínicas, Universidade Federal de Ouro Preto, Ouro Preto, MG. Address to: Dr. Girley Francisco Machado de Assis. NUPEB/UFOP. Rua Costa Sena 171, Centro, 35400-000 Ouro Preto, MG, Brasil.

Phone: $55313559-1691$

e-mail: girleyfrancisco@nupeb.ufop.br

Received in 26/02/2011

Accepted in 13/04/2011

\section{INTRODUCTION}

Chagas disease is endemic to a wide region extending from Mexico to Argentina and is one of the parasitic diseases with a major social impact in Latin America, mainly due to its high morbidity and mortality, including the occurrence of sudden death ${ }^{1}$. In the past decades, Trypanosoma cruzi infection has been increasingly found in non-endemic countries, such as the United States, Canada, Japan, Australia, and many European countries, mainly due to increasing population mobility ${ }^{2}$.

Chagas disease has two successive phases: the acute and the chronic phase, with different clinical forms referred to as asymptomatic (indeterminate form (IND)) for many years throughout the lifetime of the majority of infected people. In approximately $30 \%$ of patients, the disease evolves to cardiac (CARD), digestive (DIG), and/or mixed clinical forms ${ }^{3}$.

During the acute phase or during reactivation due to immunosupression, the diagnosis is carried out by the direct detection of circulating parasites. However, during the chronic phase, because of low parasitemia, the diagnosis is performed mainly by serological methods. The enzyme-linked immunosorbent assay (ELISA) is the most used serological technique due its high sensitivity and specificity, which is dependent on the antigen employed, the cutoff point used, and the ability to process large quantities of samples rapidly ${ }^{4}$.

However, while the serological methods used in routine diagnosis of $T$. cruzi infection are very effective in detecting infected individuals with high and medium antibody titers, they fail to detect patients with low immunoglobulin levels ${ }^{5}$. This and other limitations have been the main reason of the continuing research on the ELISA technique and its targeted antigens for the purpose of eliminating the occurrence of false-positive and false-negative results.

The sera reactivity of patients with chronic Chagas disease, human T-cell lymphotropic virus (HTLV), hepatitis B virus (HBV), hepatitis C virus (HCV), syphilis (SYP), visceral leishmaniasis (VL), 
and American tegumentary leishmaniasis (ATL) was assessed using a whole lysate and recombinant (CRA + FRA) antigen ${ }^{6}$. The recombinant ELISA showed $100 \%$ sensitivity and specificity, with no cross-reactivity with other infections. However, the conventional ELISA showed serological cross-reactivity with VL. Although the use of purified antigen preparations increased the specificity of the ELISA tests, its availability in health systems is still limited due to its higher cost.

Several authors have already studied the relationship between the intensity of serological reactivity of different IgG subclasses and Chagas disease morbidity ${ }^{7-12}$. Morgan et al detected increased levels of IgG2 in CARD patients using an ELISA test based on epimastigotes of the Brazil strain. Verçosa et al. also verified increased levels of IgG2 in CARD patients using an ELISA test based on the flagellar portion of the T. cruzi. Increased levels of IgG1 and IgG2 in all Chagas disease patients, independent of their clinical form, were found. At the same time, the highest levels of IgG2 were found in the CARD group 9 Different results using other antigenic preparations were observed and have been verified to be predominantly IgG1 and IgG3 in all studied Chagas disease patients, while IgG2 reactivity was observed in only $50 \%$ of cases $^{13}$. However, equal concentrations of IgG1, IgG2, IgG3, and IgG4 in all studied Chagas disease patients, independent of their clinical form, were verified ${ }^{7}$. Associations between other classes of immunoglobulins and clinical forms of Chagas disease have also been found. Higher levels of IgM were predominant in patients presenting the cardiac form, while higher levels of IgA were predominant in those with the digestive form ${ }^{10}$. The predominance of $\operatorname{IgA}$ was also verified in patients in the acute phase and in those with the digestive form in the chronic phase ${ }^{14}$, corroborating previous data from this team ${ }^{15}$.

Taking into account the limitations of the available serological tests for Chagas disease diagnosis and the existent controversy regarding the correlation between serological reactivity and clinical manifestations of Chagas disease, the objectives of this investigation were to develop an in-house ELISA, to propose criteria for the analysis of the reactivity of total IgG and IgG subclasses to T. cruzi antigens, and to evaluate the applicability of the in-house method in the differential diagnosis of Chagas disease and the analysis of correlations with the clinical forms of the disease.

\section{METHODS}

\section{Study area}

The municipality of Berilo is located in the Jequitinhonha Valley, northeast of Minas Gerais State, Brazil (10.57’06”S; 42.27'56”W), at an altitude of $401 \mathrm{~m}$. The territorial area of Berilo is $586 \mathrm{~km}^{2}$, and its total population is 17,549 inhabitants, $77 \%$ of whom live in the rural area. The economic activity of the population is mainly based on agriculture and livestock production.

\section{Patients and sera samples}

Sixty Chagas disease patients $(\mathrm{CH})$ were selected in Berilo municipality, Minas Gerais State, Brazil, from March to November 2006. All patients presented positive serological results in the diagnosis of T. cruzi infection (ELISA and indirect haemaglutination assay (IHA)). The clinical evaluation was performed through anamnesis, physical examination, conventional electrocardiogram (ECG), thorax X-ray, and esophagus and colon barium-contrast $\mathrm{X}$-rays ${ }^{16}$. Additionally, echocardiographic evaluation of all patients was performed using a Cypress portable device with unidimensional, bidimensional, continuous, pulsate and color Doppler adapted for tissue Doppler imaging. A multifrequency transductor, at 3.0 to $3.5 \mathrm{MHz}$, with simultaneous electrocardiography registration was used. A standard echocardiography protocol was performed assessing the following variables: cardiac dimensions, left ventricular systolic and diastolic functions, and the global and segmental contractibility of the left ventricle. The patients were clinically classified according to the Brazilian Consensus on Chagas Disease ${ }^{17}$. Those with neither Chagas disease symptomatology nor alterations in the physical examination, ECG, echocardiography, thorax X-ray, and esophagus and colon contrast X-rays were classified under indeterminate form (IND) $(n=15$, including 9 males and 6 females, with ages ranging from 13 to 57 years). The CARD group ( $\mathrm{n}=30$, including 5 males and 25 females, with ages ranging from 25 to 60 years) included patients with any type of cardiac alteration detected through either referred symptoms or detected signs in the physical examination, ECG, and echocardiography; and without any alteration in the thorax X-ray, and esophagus and colon contrast X-rays. Another group of Chagas disease patients simultaneously presenting digestive alterations detected by esophagus and colon contrast X-rays, with or without cardiac alterations, were classified as digestive/mixed (DIG/Mix) group $(\mathrm{n}=15$, including 6 males and 9 females, with ages ranging from 37 to 59 years).

All patients in this study were integrated into the clinical assistance program of the Chagas disease project of Jequitinhonha Valley, Minas Gerais, and received continuous assistance when necessary.

A control group of noninfected individuals (NI) ( $n=15$, including 6 males and 9 females, with ages ranging from 17 to 54 years) was selected from the local population of Berilo municipality, Minas Gerais, Brazil; all of them presented negative results of Chagas disease serological diagnosis. These subjects were also clinically evaluated through anamnesis, physical examination, conventional ECG, and chest X-ray.

Sera samples from patients infected with other relevant infectiousparasitic diseases were also included in this study. These patients were categorized as non-chagasic patients $(\mathrm{NCH})$ and further subgrouped as patients infected with human immunodeficiency virus (HIV) $(\mathrm{n}=8$, including 6 males and 2 females, with ages ranging from 19-60 years), hepatitis $\mathrm{C}$ virus ( $\mathrm{HCV})(\mathrm{n}=8$, including 7 males and 1 female, with ages ranging from 18 to 54 years), syphilis (SYP) ( $n=8$, including 4 males and 4 females, with ages ranging from $18-58$ years), visceral leishmaniasis (VL) ( $\mathrm{n}=7$, including 5 males and 2 females, with ages ranging from 20-67 years), and American tegumentary leishmaniasis (ATL) $(\mathrm{n}=8$, including 5 males and 3 females, with ages ranging from 16-54 years). All patients with other infectious-parasitic diseases were residents in Belo Horizonte (HIV, HCV, and SYP), Montes Claros (VL), and Caratinga (ATL), all situated in State of Minas Gerais, Brazil.

\section{In-house ELISA for evaluation of anti-Trypanosoma cruzi total IgG and IgG subclasses}

The in-house ELISA methodology modified ${ }^{18}$ proceeded as follows. Briefly, each well of the ELISA plate was sensitized overnight at $4^{\circ} \mathrm{C}$ with $100 \mu \mathrm{L}$ of T. cruzi Y-strain antigen $(4.5 \mu \mathrm{g} / \mathrm{mL})$ prepared by alkaline extraction. Following washing steps and blocking procedures with phosphate-buffered saline (PBS) supplemented with $10 \%$ fetal bovine serum, $100 \mu \mathrm{L}$ of serial dilution from each sera sample $(1: 10,1: 20,1: 40,1: 80,1: 160,1: 320,1: 640$, and 1:1,280) was added to selected wells and the plate incubated for $45 \mathrm{~min}$ at 
$37^{\circ} \mathrm{C}$. After washing with PBS $0.05 \%$ Tween $20,100 \mu \mathrm{L}$ of mouse anti-human anti-IgG and anti-IgG subclass biotinilated antibodies (Sigma, St. Louis, USA) were added to specific wells and the plate incubated for $45 \mathrm{~min}$ at $37^{\circ} \mathrm{C}$. The standardization of each conjugate dilution was previously performed using a range of serial dilutions (anti-IgG - 1:7,500, 1:10,000, 1:15,000, and 1:25,000; anti-IgG1 $1: 500,1: 1,000,1: 2,500$, and 1:5,000; anti-IgG2 - 1:2,500, 1:5,000, and 1:7,500; and anti-IgG3 - 1:1,000 and 1:2,000) to assay positive and negative sera samples at $1: 80$ dilution. The anti-IgG4 conjugate was also titrated and did not show any reactivity; therefore, it was excluded from further analysis (data not shown). The selected dilution for each conjugate was established considering the highest difference between the median OD for positive and negative samples (delta median - $\Delta$ ) (Figure 1). By this approach, 1:10,000, 1:500, $1: 2,500$, and 1:1,000 dilutions were selected for anti-IgG, anti-IgG1,
anti-IgG2, and anti-IgG3 conjugates, respectively (Figure 1). Following incubation and washing procedures with PBS 0.05\% Tween $20,100 \mu \mathrm{L}$ of streptavidin/horseradish peroxidase (HRP) (Sigma, St. Louis, USA) was added to all wells and the plates incubated for $45 \mathrm{~min}$ at $37^{\circ} \mathrm{C}$. The standardization of the streptavidin/HRP dilution was previously performed using a range of serial dilutions (1:500, $1: 1,000$, and $1: 2,500)$, and the dilution 1:1,000 was selected as the best condition to segregate positive and negative samples. After incubation and washing procedures with PBS 0.05\% Tween 20, $100 \mu \mathrm{L}$ of substrate solution (1mg of orthophenylenediamine (OPD), $3 \mathrm{~L}$ of $\mathrm{H}_{2} \mathrm{O}_{2}(30 \mathrm{vol})$, and $15 \mathrm{~mL}$ of citrate buffer $)$ was added to each well and the plates incubated for $5 \mathrm{~min}$ at $37^{\circ} \mathrm{C}$. Following incubation, the enzymatic reaction was stopped by adding $32 \mu \mathrm{L}$ of $2.5 \mathrm{M} \mathrm{H}_{2} \mathrm{SO}_{4}$ solution to each well. The optical density (OD) was determined using a 490nm filter (SOFTMAX ${ }^{\circledR}$ PRO 4.0-Life Sciences Edition).

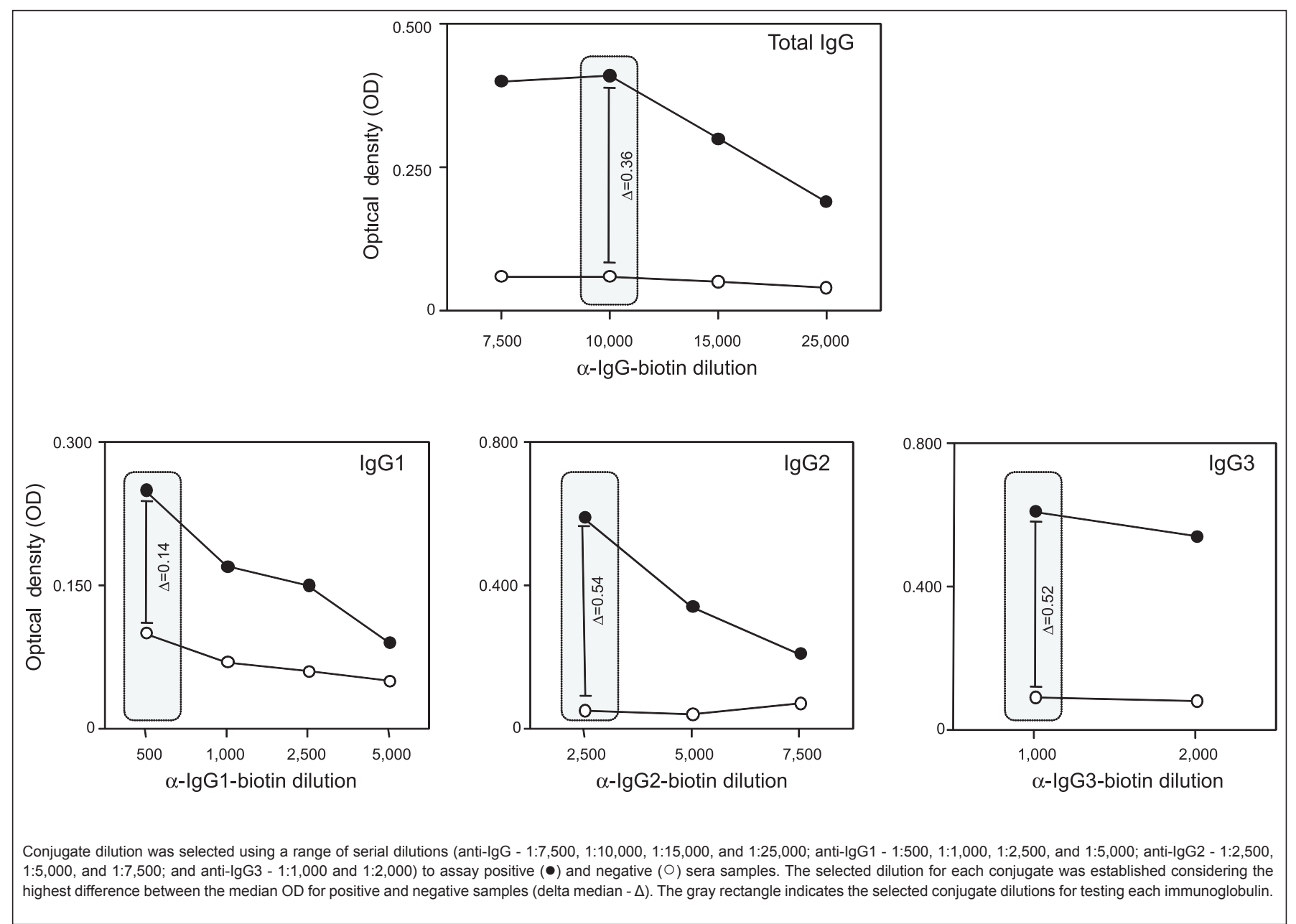

FIGURE 1 - Standardization of in-house ELISA method for the detection of total IgG and IgG subclasses (IgG1, IgG2, and IgG3) using Trypanosoma cruzi Y-strain alkaline antigen.

\section{Data interpretation and statistical analysis}

The best sera dilution to segregate the IgG reactivity of sera samples from T. cruzi-infected patients and NI was initially established along the titration curve, considering the highest difference between the median OD for positive and negative samples (delta median $-\Delta$ ) (Figure 2). Thereafter, the sera dilution that was shown to have higher performance by the construction of the receiver operating characteristic curve (ROC) using the Medcalc statistical program (Broekstraat, Mariakerke, Belgium) was selected and identified as the most useful condition to discriminate chagasic patients from NI individuals. The cutoff point to discriminate serological reactivity was selected for each immunoglobulin (total IgG, IgG1, IgG2, and IgG3), taking into account the ROC curve parameters, including the specificity, sensitivity index, and area under the curve (AUC) (Figure 2). The selected conditions were used to evaluate the IgG reactivity of sera samples from patients with other relevant infectious-parasitic diseases (Figure 3). The data were further analyzed with the two-graph receiver operating characteristic (TG-ROC) using the CMDT software package ${ }^{19}$. 


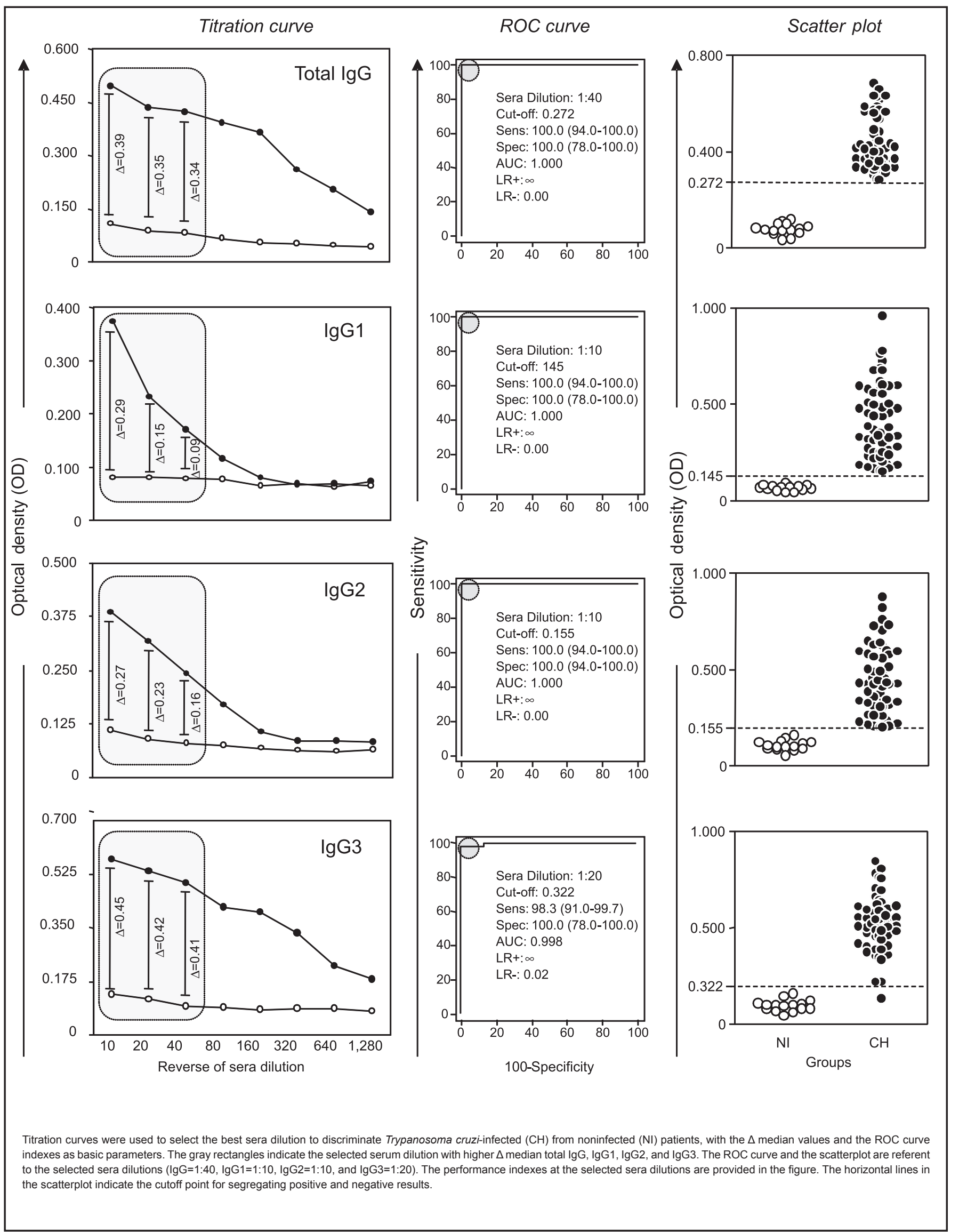

FIGURE 2 - Analysis of in-house ELISA reactivity of total IgG and IgG subclasses in sera samples from Chagas disease patients (CH •) and noninfected controls (NIO) using Trypanosoma cruzi Y-strain alkaline antigen. 


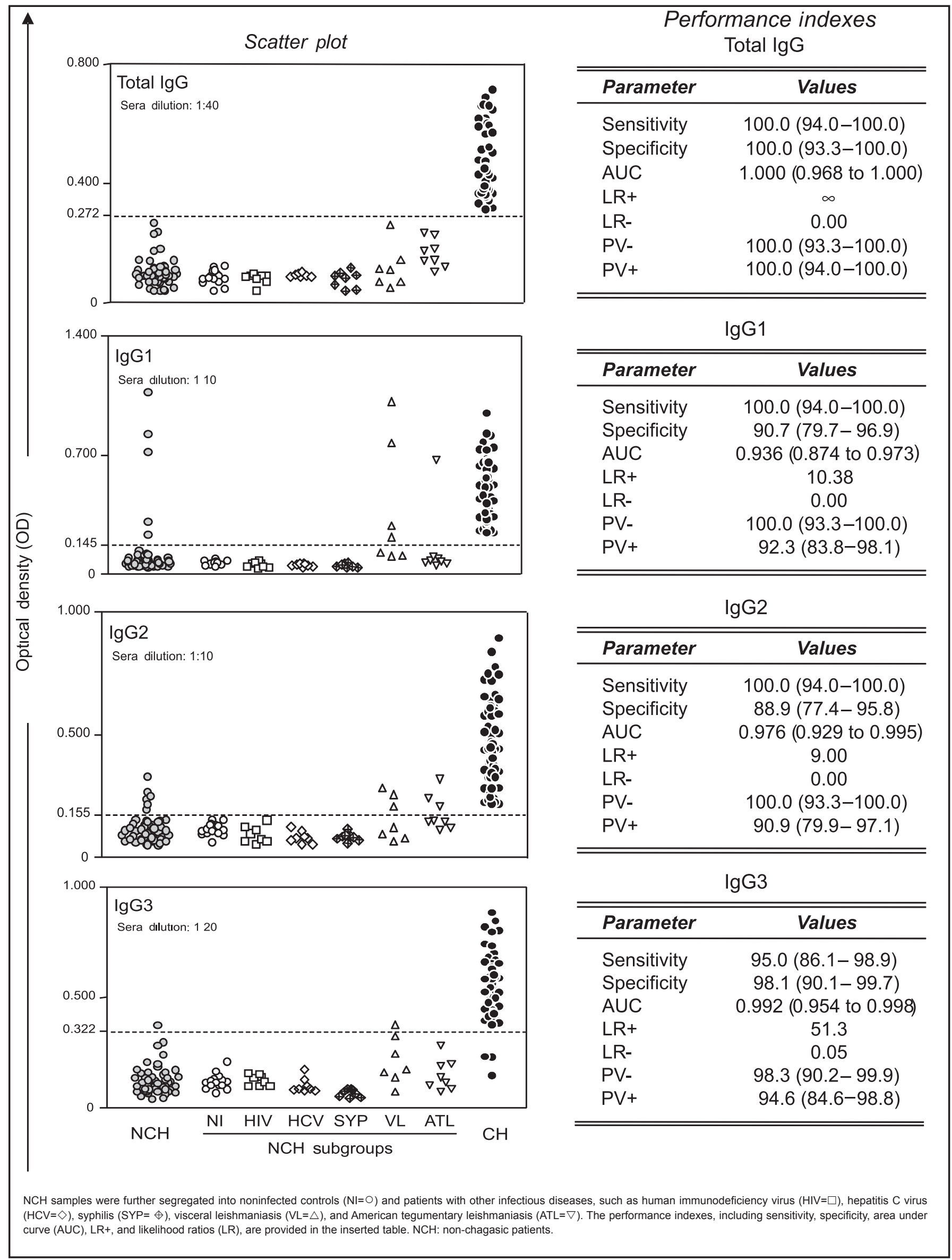

FIGURE 3 - Scatterplots of in-house ELISA reactivity at selected serum dilutions for total IgG (1:40) and IgG subclasses (IgG1=1:10, IgG2=1:10, IgG3=1:20) in sera samples from Chagas disease patients $(\mathrm{CH}=)$ and non-chagasic samples $(\mathrm{NCH}=\mathrm{O})$ using Trypanosoma cruzi $\mathrm{Y}$-strain alkaline antigen. 
This method reads valid pairs of sensitivity and specificity considering pre-assigned cutoff values and allows the selection of the best condition for data interpretations. The cutoff of the sensitivity and specificity intersection point $\left(\theta_{0}\right)$ provides equal test indices. According to Greiner et al., whenever the value of $\theta_{0}$ is lower than a preselected accuracy level (90\% or $95 \%$ ), two cutoff values are selected to represent the limits of an intermediate range (IR). The IR can be considered as a borderline range for the clinical interpretation of the test results. The selection of cutoff values was corroborated by graphical displays of logarithmically transformed likelihood ratios (LR) (LR+=Se/ $(1-\mathrm{Sp})$ and $\mathrm{LR}-=(1-\mathrm{Se}) / \mathrm{Sp})$. The Efficiency and Youden $(\mathrm{J})$ indices were also presented to support the cutoff selection (Figure 4).
Differences in the OD median values of IgG reactivity between different clinical groups of Chagas disease patients (IND, CARD, and DIG/Mix) were evaluated by the Kruskal-Wallis test, followed by Dunn's test, using the GraphPad Prism software (San Diego, CA, USA). Statistical significance in all cases was considered at $\mathrm{p}<0.05$ (Figure 5).

\section{Ethical considerations}

All volunteers were included in the study after they had read and signed an informed consent form approved by the Ethics Committee of the Centro de Pesquisas René Rachou (CPqRR), FIOCRUZ, Belo Horizonte, Minas Gerais, Brazil (Process 0007/02).

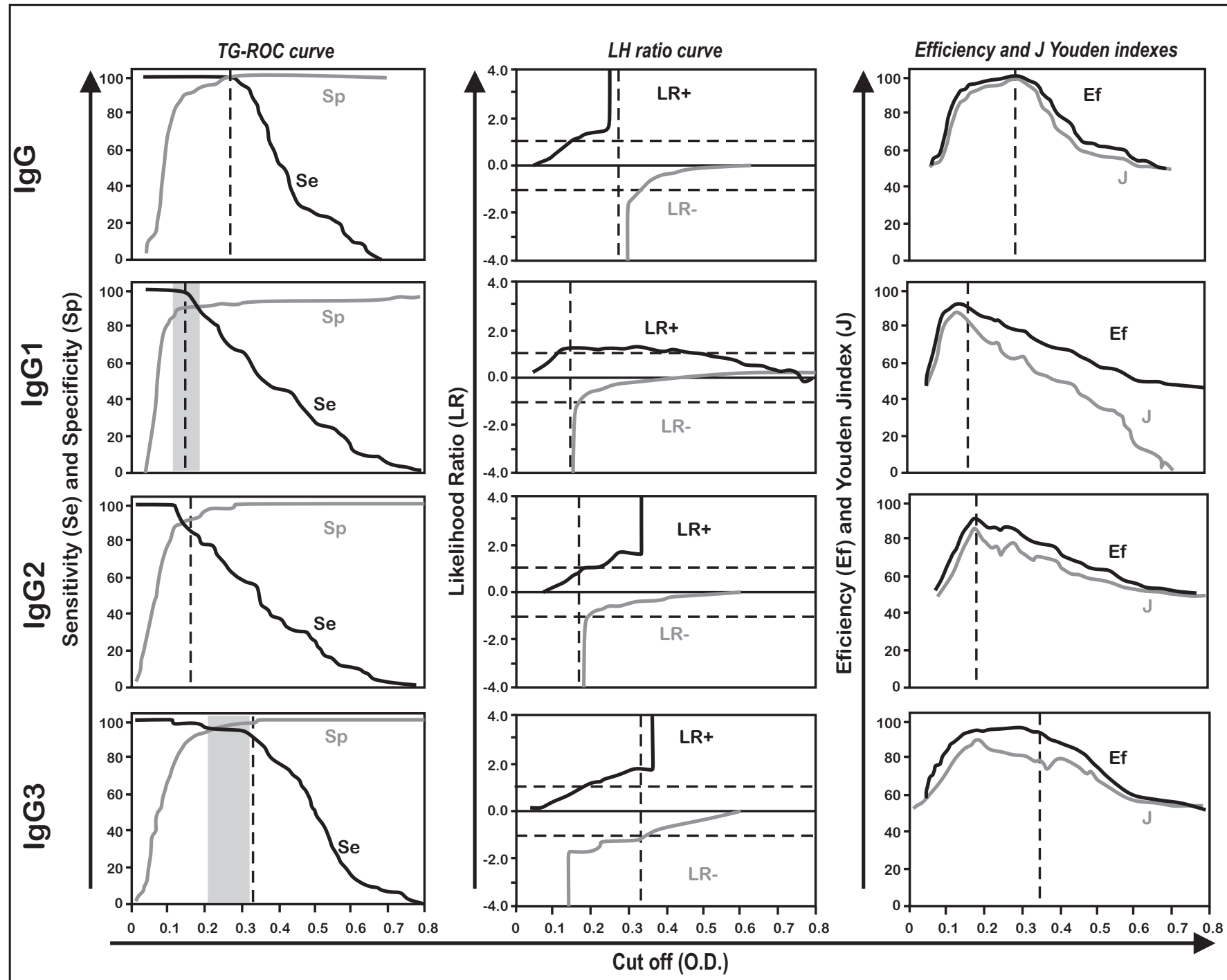

The TG-ROC curves indicate valid pairs of sensitivity (se) and specificity (sp) considering pre-assigned cutoff values, confirming the selection of the best cutoff edge for data interpretations. The gray rectangles indicate the intermediate range (IR), considered as a borderline range for the interpretation of results. The likelihood ratio curve (LR+ and LR-) corroborates the selection of the cutoff edges. The Efficiency (Ef) and Youden (J) indexes are also displayed to support the cutoff selection. The dotted rectangles highlight the cutoff ranges with the most outstanding performances.

FIGURE 4 - Analysis of in-house ELISA reactivity at selected serum dilutions for total IgG (1:40) and IgG subclasses (IgG1=1:10, IgG2=1:10, IgG3=1:20) in sera samples from Chagas disease patients and non-chagasic samples using Trypanosoma cruzi Y-strain alkaline antigen. 


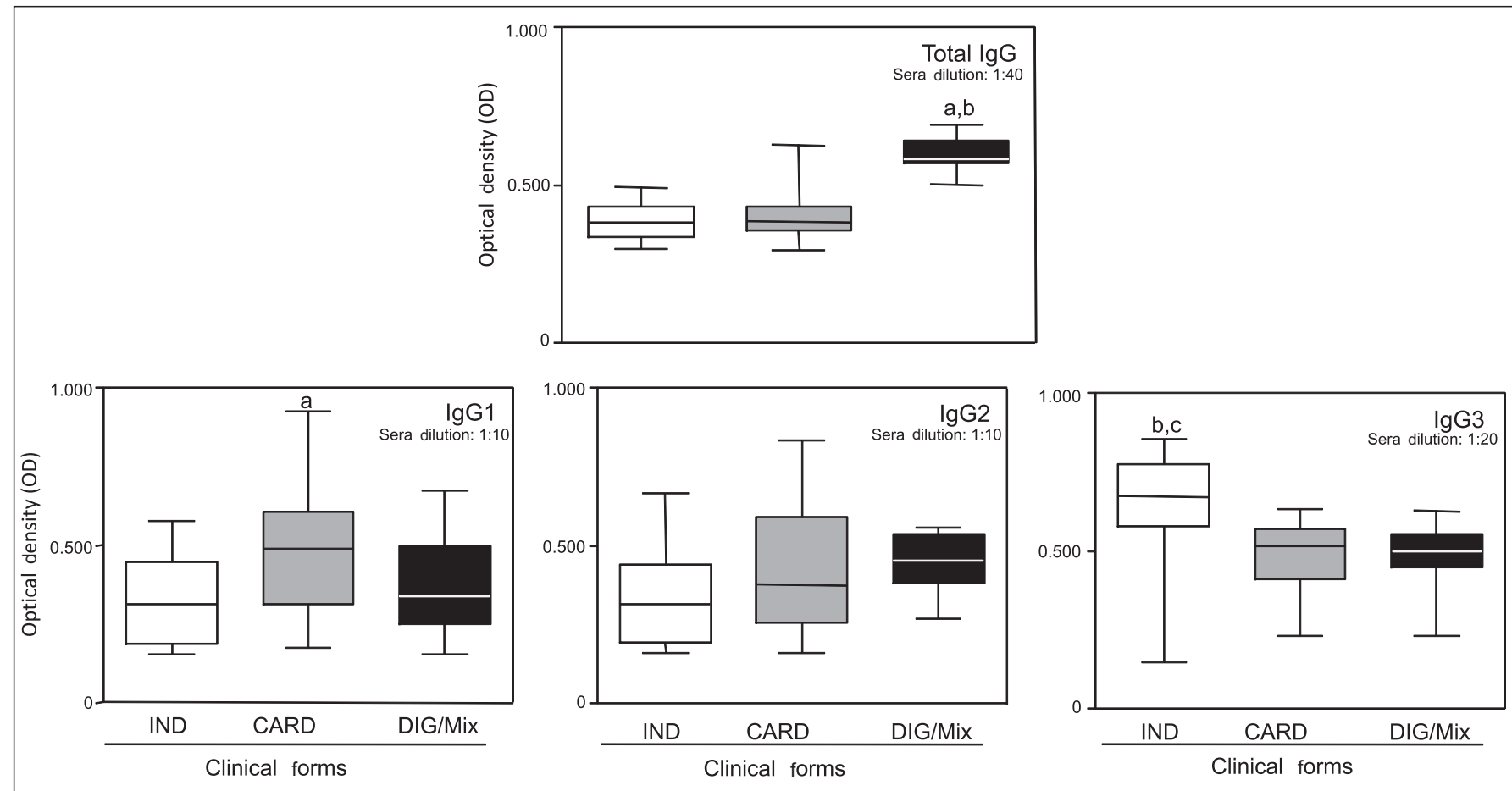

Data are presented in box plot format, highlighting the mean reactivity values with a line crossing the boxes. The minimum and maximum values are represented by stretching lines. Letters $a, b$, and $c$ indicate significant differences at $p<0.05$ among IND, CARD and DIG/Mix groups.

FIGURE 5 - Analysis of in-house ELISA reactivity at selected serum dilutions for total IgG (1:40) and IgG subclasses (IgG1=1:10, IgG2=1:10, IgG3=1:20) in sera samples from Chagas disease patients from Berilo municipality, Jequitinhonha Valley, State of Minas Gerais, Brazil, categorized under indeterminate (IND), cardiac (CARD), or digestive/mixed (DIG/Mix) group.

\section{RESULTS}

\section{Discrimination between Trypanosoma cruzi-infected and noninfected individuals by the differential reactivity of total IgG and IgG subclasses}

Upon its standardization, the in-house ELISA (Figure 1) method was used to evaluate the reactivity of total $\operatorname{IgG}$ and $\operatorname{IgG}$ subclasses, with the aim of discriminating T. cruzi-infected $(\mathrm{CH})$ from noninfected individuals (NI). During the standardization procedure, the best dilution of second-step reagents to be applied for diagnosis purposes was chosen by considering the delta median ( $\Delta$ median) between positive $(\mathrm{CH})$ and negative samples $(\mathrm{NI})$, as well as the ROC curve indexes for each immunoglobulin. Using a similar approach to select the best sera dilution to be applied for diagnosis purposes, our findings demonstrated that only the first three serum dilutions presented an outstanding ability to discriminate the reactivity of total IgG, and IgG1, IgG2, and IgG3 subclasses along the titration curve, to segregate patients with $T$. cruzi infection $(\mathrm{CH})$ from noninfected controls (NI). The data analysis demonstrated distinct $\Delta$ median values for $\operatorname{IgG}(0.39,0.35$, and 0.34$), \operatorname{IgG} 1(0.29,0.15$, and $0.09), \operatorname{IgG} 2(0.27,0.23$, and 0.16$)$, and $\operatorname{IgG} 3(0.45,0.42$, and 0.41$)$ at serum dilutions of $1: 10,1: 20$, and $1: 40$, respectively. Although the $\Delta$ median value was higher at 1:10 dilution for all $\operatorname{IgG}$ reactivity, the selected sera dilution also took into account the best performance in the ROC curve analysis including sensitivity, specificity, and AUC. Using these criteria, the sera dilution 1:40 was elected for IgG, 1:10 for IgG1 and IgG2, and 1:20 for IgG3 (Figure 2).
The use of the cutoff edge indicated by the ROC curve analysis revealed outstanding performance indexes for $\operatorname{IgG}$, IgG1, and IgG2, with $100 \%$ sensitivity and specificity, and $\mathrm{AUC}=1.0$. Moreover, the LR ratio analysis by the ROC curve further confirmed these indicators (Figure 2). The analysis of IgG3 demonstrated indexes with lower power to discriminate the clinical groups $\mathrm{CH}$ and $\mathrm{NCH}$, with 98.3\% sensitivity and AUC=0.998 (Figure 2).

\section{Discrimination between Trypanosoma cruzi infection and other infectious diseases by the differential reactivity of total IgG and IgG subclasses}

Considering the excellent performance of the in-house ELISA in discriminating chagasic patients from noninfected controls, an additional analysis was subsequently performed to evaluate the reactivity of sera samples from chagasic patients in parallel with samples from noninfected controls and also from patients with other relevant infectious-parasitic diseases, including HIV, HCV, SYP, VL, and ATL.

The analysis of total IgG at serum dilution 1:40 was able to discriminate patients with T. cruzi infection from patients with the other studied infectious diseases (HIV, HCV, SYP, VL, and ATL) and NI individuals, with maximum performance indexes, including $100 \%$ sensitivity and specificity, AUC of 1.0, and LR+ and LR-values indicating infinite probability of finding a positive result for $\mathrm{CH}$ and a negative result for $\mathrm{NCH}$ (Figure 3).

Although the analysis of $\operatorname{IgG} 1$ at serum dilution 1:10 showed $100 \%$ sensitivity, indicating that all $\mathrm{CH}$ presented a positive result, the low specificity (90.7\%) indicated the presence of cross-reactivity in 5 out of 54 tested samples, including $7.4 \%$ false-positive results due to 
four VL samples and $1.9 \%$ due to one ATL sample. The AUC=0.936, $\mathrm{LR}+=10.38$, and $\mathrm{LR}=0$ indicated lower performance as compared with total IgG (Figure 3).

The evaluation of IgG2 reactivity at serum dilution $1: 10$ also showed $100 \%$ sensitivity but also revealed lower specificity ( $88.9 \%)$, with cross-reactivity in $11.1 \%$ of the tested samples, due to the existence of false-positive results in three VL and three ATL samples. The AUC $=0.976, \mathrm{LR}+=9$, and LR- $=0$ indicated lower performance as compared with total IgG (Figure 3).

The analysis of $\operatorname{IgG} 3$ reactivity at serum dilution 1:20 demonstrated low sensitivity (95\%), with three out of 60 patients with T. cruzi infection showing OD values below the cutoff point. The specificity of $98.1 \%$ was due to cross-reactivity observed in one VL sample. The AUC $=0.992$ showed high accuracy. Although the $L R+=51.3$ indicated that a positive result has higher probability of belonging to the T. cruziinfected population as compared with $\mathrm{NCH}$, the $\mathrm{LR}-=0.05$ showed borderline performance in identifying negative results (Figure 3 ).

Considering the good performance of the in-house ELISA when applied to the diagnosis of Chagas disease and aiming to validate our findings with supplementary performance analysis, the data were further evaluated using novel parameters, including the TG-ROC, the LR ratio in log base graphs, and the simultaneous analysis of the Efficiency and Youden indexes (Figure 4). In the first approach, valid pairs of sensitivity and specificity were read for pre-assigned cutoff values directly from the TG-ROC plots $^{19}$. The TG-ROC analysis showed that the in-house ELISA used to detect total IgG reactivity did not present any intermediate range (IR) that can be considered a borderline range for the clinical interpretation of test results, therefore indicating that the method displayed $100 \%$ valid data with no incorrect results. These findings were also confirmed by the maximum LR+ and LR-, and the Efficiency and Youden index analysis (Figure 4). Together, these analyses showed that the anti-T. cruzi IgG detected by the in-house ELISA at sera dilution 1:40 using the cutoff point $=0.272$ was adequate to confirm the diagnosis of Chagas disease and exclude non-chagasic samples (Figure 4).

Although the analysis of IgG2 performance also evidenced the absence of an intermediate range in the TG-ROC graph, as reported in the previous analysis (Figure 3), it was not possible for the IgG2 reactivity to conclusively discriminate $T$. cruzi-infected individuals from non-chagasic patients, considering the cross-reactivity with sera from VL and ATL patients.

The most unsatisfactory results were found in the analysis of IgG1 and IgG3 reactivity. The analyses of IgG1 and IgG3 anti-T. cruzi reactivity revealed the presence of an intermediate range, corresponding to the existence of gray zones that do not guarantee a clear discrimination between T. cruzi-infected and noninfected individuals. Moreover, the LR analysis of IgG1 reactivity did not show outstanding performance. Therefore, together with the previous analysis (Figure 3), these findings did not recommend the use of the IgG1 and IgG3 detected by the in-house ELISA proposed in this study as a valuable method for diagnosis of Chagas disease (Figure 4).

\section{Applicability of the in-house ELISA in analyzing total IgG and IgG subclasses reactivity and monitoring Chagas disease morbidity}

The analysis of IgG reactivity at serum dilution 1:40 revealed that the DIG/Mix group presented significantly higher OD reactivity as compared with the IND and CARD groups (Figure 5). On the other hand, the IgG1 reactivity at serum dilution 1:10 and the IgG3 reactivity at serum dilution 1:20 demonstrated that the median OD for the IND group was significantly lower as compared with that for the CARD group (Figure 5). No significant differences were observed in IgG2 reactivity at sera dilution 1:10 among the distinct clinical groups evaluated (Figure 5).

\section{DISCUSSION}

In this study, an in-house ELISA method was standardized to better determine the reactivity of total IgG, and IgG1, IgG2, and IgG3 subclasses in T. cruzi-infected and noninfected patients. Additionally, the in-house ELISA technique was applied to verify the reactivity of these immunoglobulin classes and subclasses in groups of individuals with T. cruzi infection and other relevant infectious-parasitic diseases (HIV, HCV, SYP, VL, and ATL). Initially, after the standardization procedures to define the best dilutions of the second step reagents (Figure 1), the goal of the study was to verify the best serum dilutions able to discriminate Chagas disease patients from the other selected groups. Our findings demonstrated that only total IgG reactivity showed no cross-reactivity with sera from patients without T. cruzi infection. However, the IgG1 and IgG2 analysis showed crossreactivity with sera samples from VL and ATL patients. IgG2 showed higher cross-reactivity with sera samples from ATL as compared with IgG1 and IgG3. This finding is in agreement with previous reports showing that T. cruzi-infected patients presented high levels of crossreactivity of IgG2 with Leishmania braziliensis antigens ${ }^{20}$.

The cross-reactivity between anti-Leishmania and anti-T. cruzi IgG2 may explain these data. Although IgG3 showed lower crossreactivity with other diseases, with only one false-positive result, the performance of this subclass was not validated due to the low sensitivity index. These findings agreed with the data in the literature demonstrating the existence of shared antigens between T. cruzi and Leishmania sp., such as carbohydrate epitopes ${ }^{20-23}$. On the other hand, the use of crude antigens in this in-house ELISA may explain this cross-reactivity since the same phenomenon was not observed in the sera of patients with VL, ATL, SYP, HIV, and HCV when CRA+FRA recombinant antigens were used ${ }^{6}$. Thus, the discovery and availability of purified antigens or antigenic fractions from T. cruzi with higher specificity and similar sensitivity to avoid cross-reactions among these and other infections may represent a next step in further investigations to validate in-house ELISA methods. Nevertheless, the present study demonstrated that the detection of total IgG using an in-house ELISA technique with T. cruzi crude alkaline antigenic extracts, as proposed by our group at the Laboratório de Doença de Chagas of the Universidade Federal de Ouro Preto, represent a valuable tool for the diagnosis of T. cruzi infection, with outstanding performance indexes. The major changes performed in the present investigation as compared with the original methodology proposed ${ }^{18}$ included the use of biotinilated second-step reagents, the use of serial dilutions for all tested serum samples, and a standardized incubation temperature of $37^{\circ} \mathrm{C}$. The use of biotinilated reagents may lead to increased performance of this in-house ELISA due to the reagents' ability to expand the detection capacity of the system from that in the original methodology. Moreover, these reagents require a shorter development time $(5 \mathrm{~min})$ as compared with that in the original method $(15 \mathrm{~min})$, which may reduce the occurrence of cross-reactivity and false-positive results. 
The second goal of this study was to verify whether the reactivity of total IgG and the different IgG subclasses detected by the in-house ELISA was associated with the different clinical forms of the disease. Firstly, this analysis showed that total IgG and IgG3 showed the highest OD values in all patients, followed by IgG2 and IgG1. The total IgG was higher in patients from the DIG/Mix group than in the other clinical groups. The total IgG reactivity was significantly different among the different clinical forms of the disease and presented a positive correlation with the existence of clinical manifestations of the disease. These results agree with a study ${ }^{24}$ that used the IHA based on T. cruzi antigens of the $\mathrm{Y}$ strain, which verified that the total IgG levels were slightly higher in patients from the State of Goiás, Brazil, with severe cardiomyopathy and digestive manifestations. However, the same authors did not observe the same correlation when they used an ELISA and a complement fixation reaction (CFR), indicating that the biological meaning of these findings is not understood. Patients with chagasic cardiopathy from Virgem da Lapa, the municipality next to Berilo, presented higher total IgG reactivity independent of the use of different serological tests (conventional ELISA, recombinant ELISA, IHA, and indirect immunofluorescence (IIF)) and antigenic preparations ${ }^{25}$. Differently, no association between levels of IgG and clinical manifestations of Chagas disease was observed when 225 patients with different clinical forms of Chagas disease, 121 patients with other diseases (SYP, toxoplasmosis, kalazar, mucocutaneous leishmaniasis, connective tissue diseases, malaria, rubeola, and Pemphigus foliaceus infection), and 160 healthy individuals were evaluated using antigens at different stages of the parasite life cycle (Y strain), and ELISA, IHA, CFR, and IIF techniques ${ }^{14}$. Similarly, no correlation was observed by Cordeiro et $\mathrm{al}^{8}$, when they evaluated other groups of chagasic patients from Argentina and State of Minas Gerais, Brazil.

The analysis of the IgG1 subclass demonstrated lower reactivity in the IND group as compared with the CARD group. This finding is discrepant to those previously reported by several other authors $^{7-10,13,26-27}$. Only at serum dilution 1:10 was a significant difference in the OD of IgG1 observed between the IND and CARD groups. No correlation (neither positive nor negative) with morbidity of Chagas disease was observed with the IgG1 subclass, possibly due to the low $\mathrm{OD}$ values observed. The results were different from those found by other authors who, even when using different serological tests, verified IgG1 predominance over the other IgG subclasses. The measure of $\operatorname{IgG1}$ was also suggested as a prognosis marker for Chagas disease since the level was found to be significantly higher in patients with the indeterminate form of the disease when compared with the other clinical groups ${ }^{8}$. In fact, we do not have a convincing explanation for the obtained results regarding IgG1.

The evaluation of IgG2 did not reveal a direct association with cardiac involvement, in contrast to other authors, who reported higher reactivity of this IgG2 subclass in patients with different levels of cardiopathy ${ }^{9-10,12}$. On the other hand, high levels of IgG3 were detected, especially in the IND group as compared with the CARD and DIG/ Mix groups. However, several studies considering the IgG3 subclass did not find the same association with higher levels of this immunoglobulin in all patients, independent of the clinical form of the disease $\mathrm{s}^{7,10,13,26}$.

The discrepancy of these results may be especially attributed to methodological variations and the diverse antigens used in the different techniques. Additionally, other variables regarding the selected casuistry may be involved, such as the geographical origin of the patients and consequent predominant T. cruzi population, and the length of the infection ${ }^{7-10,12-13,26}$.
Finally, the authors believe that this type of study is of great importance because in-house ELISA methods, when well established, may serve as important tools for the diagnosis and prognostic study of Chagas disease, especially in developing countries, where the low availability of financial support for research may represent a remarkable limitation for population serological surveys.

\section{ACKNOWLEDGMENTS}

We would like to thank the Berilo municipality for the facilities offered for this work. MTB, ATC and OAMF thank Conselho Nacional de Desenvolvimento Científico e Tecnológico for fellowships (PQ).

\section{CONFLICT OF INTEREST}

The authors declare that there is no conflict of interest.

\section{FINANCIAL SUPPORT}

Fundação de Amparo à Pesquisa de Minas Gerais (FAPEMIG) and Conselho Nacional de Desenvolvimento Científico e Tecnológico (CNPq).

\section{REFERENCES}

1. Pan American Health Organization. Estimación cuantitativa de la enfermedad de Chagas en las Américas. Montevideo, Uruguay: OPS/HDM/CD/425-06; 2006. p. 1-28.

2. Schmunis GA. Epidemiology of Chagas disease in non-endemic countries: the role of international migration. Mem Inst Oswaldo Cruz 2007; 102 (suppl 1):75-85.

3. Prata A. Clinical and epidemiological aspects of Chagas disease. Lancet Infect Dis 2001; 1:92-100.

4. Bern C, Coura JC, Goldenberg S, Guhl F, Junqueira ACV, Lorca M, et al International meeting: new diagnostic tests are urgently needed to treat patients with Chagas disease. Médecins Sans Frontières. Campaign for Access to Essential Medicines. Technical Report. Rev Soc Bras Med Trop 2008; 41:315-319.

5. Zarate-Blades CR, Bladés N, Nascimento MS, Silveira JF, Umezawa ES. Diagnostic performance of tests based on Trypanosoma cruzi excreted-secreted antigens in an endemic area for Chagas'disease in Bolivia. Diagn Microbiol Infect Dis 2006; 57:229-232.

6. Gomes YM, Pereira VRA, Nakazawa M, Rosa DS, Barros MNDS, Ferreira AGP et al. Serodiagnosis of chronic Chagas infection by using EIERecombinantChagas-Biomanguinhos kit. Mem Inst Oswaldo Cruz 2001; 96:497-501.

7. Cerban FM, Gea S, Menso E, Vottero-Cima E. Chagas' disease: IgG isotypes against Trypanosoma cruzi cytosol acidic antigens in patients with different degrees of heart damage. Clin Immunol Immunopathol 1993; 67:25-30.

8. Cordeiro FD, Martins-Filho OA, RochaMOC,AdadSJ, Corrêa-OliveiraR, RomanhaAJ. Anti-Trypanosoma cruzi immunoglobulin G1 can be a useful tool for diagnosis and prognosis of humam Chagas' disease. Clin Diagn Lab Immunol 2001; 1:112-118.

9. Hernandez-Becerril N, Nava A, Reyes PA, Monteon VM. IgG subclass reactivity to Trypanosoma cruzi in chronic chagasic patients. Arch Cardiol Mex 2001; 71:199-205.

10. Morgan J, Dias JCP, Gontijo ED, Bahia-Oliveira L, Correa-Oliveira R, Colley DG et al. Anti-Trypanosoma cruzi antibody isotype profiles in patients with different clinical manifestations of Chagas' disease. Am J Trop Med Hyg 1996; 55:355-359.

11. Morgan J, Colley DG, Pinto Dias JC, Gontijo ED, Bahia-Oliveira L, Correa-Oliveira R, et al. Analyses of anti-Trypanosoma cruzi antibody isotype specificities by western blot in sera from patients with different forms of Chagas'disease. J Parasitol 1998; 84:641-643.

12. Verçosa AFA, Lorena VMB, Carvalho CL, Melo MFAD, Cavalcanti MGA Silva ED, et al. Chagas'disease: IgG isotypes against cytoplasmic (CRA) and flagellar (FRA) recombinant repetitive antigens of Trypanosoma cruzi in chronic Chagasic patients. J Clin Lab Anal 2007; 21:271-276. 
13. Solana ME, Katzin AM, Umezawa ES, Miatello CS. High specificity of Trypanosoma cruzi epimastigote ribonucleoprotein as antigen in serodiagnosis of Chagas'disease. J Clin Microbiol 1995; 33:1456-1460.

14. Primavera KSC, Umezawa ES, Peres BA, Camargo ME, Hoshino-Shimizu S. Chagas'disease: IgA, IgM, and IgG antibodies to T. cruzi amastigote, trypomastigote and epimastigote antigens in acute and in different chronic forms of the disease. Rev Inst Med Trop São Paulo 1990; 32:172-180.

15. Primavera KSC, Hoshino-Shimizu S, Umezawa ES, Peres BA, Manigot DA, Camargo ME. Immunoglobulin A antibodies to Trypanosoma cruzi antigens in digestive forms of Chagas'disease. J Clin Microbiol 1988; 26:2101-2104.

16. Rezende JM, Moreira H. Chagasic megaesophagus and megacolon. Historical review and present concepts. Arq Gastroenterol 1988; 25 (special issue):32-43.

17. Ministério da Saúde. Brazilian Consensus on Chagas Disease. Rev Soc Bras Med Trop 2005; 38 (supl 3):7-29.

18. Voller A, Bidwell DE, Bartlett A. Enzyme immunoassays in diagnostic medicine. Theory and pratice. Bull World Health Organ 1976; 53:55-65.

19. Greiner M, Sohr D, Göbel P. A modified ROC analysis for the selection of cut-off values and the definition of intermediate results of serodiagnostic tests. J Immunol Methods 1995; 185:123-132.

20. Rocha RDR, Gontijo CMF, Elói-Santos S, Carvalho AT, Corrêa-Oliveira R, Marques MJ, et al. Anti-live Leishmania (Viannia) braziliensis, promastigote antibodies, detected by flow cytometry to identify active infection in American cutaneous leishmaniasis. Rev Soc Bras Med Trop 2002; 35:551-562.

21. Araújo FG. Analysis of Trypanosoma cruzi antigens bound by specific antibodies and by antibodies to related Trypanosomatids. Infect Immun 1986; 53:179-185.

22. Chiller TM, Samudio MA, Zoulek G. IgG antibody reactivity with Trypanosoma cruzi and Leishmania antigens in sera of patients with Chagas'disease and leishmaniasis. Am J Trop Med Hyg 1990; 43:650-656.

23. Lemos EM, Gomes IT, Carvalho SFG, Rocha RDR, Pissinate JF, Martins-Filho OA, et al. Detection of anti-Leishmania (Leishmania) chagasi immunoglobulin $\mathrm{G}$ by flow cytometry for cure assessment following chemotherapeutic treatment of american visceral leishmaniasis. Clin Vaccine Immunol 2007; 14:569-576.

24. Gusmão R, Rezende JM, Rassi A, Gam AA, Neva FA. Antibody levels to Trypanosoma cruzi in infected patients with and without evidence of chronic Chagas'disease. Am J Trop Med Hyg 1982; 31:452-458.

25. Zauza PL, Borges-Pereira J. Sera levels of IgG anti-Trypanosoma cruzi on evolution of the chronic chagasic cardiopathy in interval of 10 years. Rev Soc Bras Med Trop 2001; 34:399-405.

26. Michailowisky V, Luhrs K, Rocha MOC, Fouts D, Gazzinelli RT, Manning JE. Humoral and cellular immune responses to Trypanosoma cruzi-derived paraflagellar rod proteins in patients with Chagas disease. Infect Immun 2003; 71:3165-3171.

27. Motran CC, Serra HM, Gea SE, Vullo CM, Vottero-Cima E. Antibody isotypes profiles against Trypanosoma cruzi acidic antigens in two Amerindian populations from a Chagas'disease endemic area. Acta Trop 1994; 58:105-114. 